\title{
Pharmacokinetics of S-1 and CYP2A6 genotype in Japanese patients with advanced cancer
}

\author{
TAKASHI HIROSE ${ }^{1,2}$, KEN-ICHI FUJTTA ${ }^{2}$, KAZUKO NISHIMURA $^{3}$, HIROO ISHIDA $^{2}$, KEISHI YAMASHITA ${ }^{2}$, \\ YU SUNAKAWA ${ }^{2}$, KEIKO MIZUNO ${ }^{2}$, KEISUKE MIWA ${ }^{2}$, FUMIO NAGASHIMA ${ }^{2}$, YUSUKE TANIGAWARA ${ }^{3}$, \\ MITSURU ADACHI ${ }^{1}$ and YASUTSUNA SASAKI ${ }^{2}$
}

${ }^{1}$ Division of Respiratory Medicine and Allergology, Department of Internal Medicine, Showa University School of Medicine, 1-5-8 Hatanodai, Shinagawa, Tokyo 142-8666; ${ }^{2}$ Department of Medical Oncology, Comprehensive Cancer Center, Saitama Medical University, 1397-1 Yamane, Hidaka, Saitama 350-1298; ${ }^{3}$ Department of Pharmacy, School of Medicine, Keio University, 35 Shinanomachi, Shinjuku, Tokyo 160-8582, Japan

Received February 24, 2010; Accepted April 30, 2010

DOI: 10.3892/or_00000889

\begin{abstract}
We developed a population pharmacokinetic (PPK) model of S-1 including the cytochrome P450 (CYP) 2 A6 genotype and then used this PPK model to assess the influence of the CYP2A6 genotype on PK parameters of S-1 and the relationship between toxicity and the individual maximum concentrations $(\mathrm{Cmax})$ or the area under the concentrationtime curve (AUC) of 5-fluorouracil (5-FU) in Japanese patients with advanced cancer. Fifty-eight patients with advanced cancer were assessed. A dose of $80 \mathrm{mg} / \mathrm{m}^{2} /$ day of S- 1 was given orally. On the basis of the CYP2A6 genotypes $\left({ }^{*} 1,{ }^{*} 4\right.$, ${ }^{*} 7$ and ${ }^{*} 9$ ), all patients were classified as having the wildtype, 1 variant allele or 2 variant alleles. The PPK model was established with plasma concentration data for tegafur (FT), 5-chloro-2,4-performed dihydroxypyridine (CDHP) and 5-FU. In patients with 2 variant alleles of $C Y P 2 A 6$, the clearance of FT was 58\% less than in patients with the wildtype or 1 variant allele. The AUC of 5-FU correlated with the AUC of CDHP, but not with the AUC of FT. Therefore, the CYP2A6 genotype did not affect the AUC of 5-FU. The individual AUC or Cmax of 5-FU did not differ significantly between patients with grade 3 or 4 toxicities and patients with grade 0-2 toxicities. In conclusion, the CYP2A6 genotype did not affect the AUC of 5-FU, although the clearance of FT was lower in patients with 2 variant alleles of CYP2A6 than in patients with the wild-type or 1 variant allele.
\end{abstract}

Correspondence to: Dr Takashi Hirose, Division of Respiratory Medicine and Allergology, Department of Internal Medicine, Showa University School of Medicine, 1-5-8 Hatanodai, Shinagawa, Tokyo 142-8666, Japan

E-mail: thirose@med.showa-u.ac.jp

Key words: CYP2A6 genotype, pharmacokinetics, S-1, cancer

\section{Introduction}

S-1 is an oral fluoropyrimidine agent that consists of tegafur (FT), which is a prodrug of cytotoxic 5-fluorouracil (5-FU) and two biochemical modulators, 5-chloro-2,4-dihydroxypyridine (CDHP) and potassium oxonate in a molar ratio of 1:0.4:1 (1). CDHP competitively inhibits dihydropyrimidine dehydrogenase (DPD), a key enzyme for 5-FU degradation, and enhances the anticancer activity of 5-FU by increasing its half-life. Potassium oxonate inhibits the phosphorylation of 5-FU in the gut by inhibiting the enzymatic pyrimidine phosphoribosyl transferase pathway and reduces the gastrointestinal toxicity of active 5-FU without interfering with its antitumor activity (2). S-1 is active against cancers of the gastric, colon, rectum, lung, pancreas and head and neck (3-5). The principal toxicities of S-1 are myelosuppression and gastrointestinal toxicity (3-5).

Because anticancer drugs have narrow therapeutic windows, interindividual variability in pharmacokinetics $(\mathrm{PK})$ due to inherited differences in metabolism and excretion may result in unpredictable toxic effects (6). If drug metabolism could be predicted in each patient, individualized doses could be administered to optimize drug exposure and minimize unacceptable toxicity. Therefore, understanding interpatient PK variability is important for optimizing anticancer treatment. A population pharmacokinetic (PPK) approach can be used to estimate the parameters of mean, interindividual and residual variability of PK of drugs and to identify the factors that cause interpatient variability. The PPK model of S-1 in Western cancer patients have been described by Comets et al (7).

However, S-1 tolerance has ethnic differences. The maximum tolerated dose is substantially lower in Western patients than in Japanese patients, and for comparable S-1 doses, the area under the concentration-time curve (AUC) seem to be higher for 5-FU and lower for FT in Western patients (8-11). Additionally, PPK model by Comets et al did not include the cytochrome P450 (CYP) 2 A6 genotype. The biotransformation of FT to 5-FU is catalyzed by the hepatic drug-metabolizing enzyme CYP2A6 (12). CYP2A6 is a polymorphic enzyme 
whose activity shows considerable interindividual variability (13). $C Y P 2 A 6^{*} 1$ is defined as the wild-type allele. $C Y P 2 A 6^{*} 4$ causes a lack of enzymatic activity $(14,15)$. $C Y P 2 A 6^{*} 7$ is a single nucleotide polymorphism $(1412 \mathrm{~T} \rightarrow \mathrm{C})$ causing an amino acid change (I471T) that decreases enzymatic activity (16). $C Y P 2 A 6^{*} 9$ has a $-48 \mathrm{~T} \rightarrow \mathrm{G}$ nucleotide substitution in the TATA box of the 5'-flanking region of the $C Y P 2 A 6$ gene which reduces the expression levels of $C Y P 2 A 6$ mRNA and protein in human livers (17). Therefore, $C Y P 2 A 6$ genetic variability may be an important factor in the incidence or severity of S-1 induced toxicity. Moreover, these CYP2A6 polymorphisms are seen more frequently in the Japanese than in the Caucasians $(13,14)$.

To our knowledge, a PPK model including the CYP2A6 genotype for S-1 has not previously been reported. Therefore, we developed a PPK model of S-1 including the CYP2A6 genotype and then used this PPK model to assess the influence of the CYP2A6 genotype on PK parameters of S-1 and the relationship between toxicity and the individual maximum concentrations (Cmax) or AUC of 5-FU in Japanese patients with advanced cancer.

\section{Materials and methods}

Study participants. The criteria for study entry were: i) histologically or cytologically confirmed solid malignancy; ii) metastatic or recurrent disease; iii) age of 20 years or older; iv) Eastern Cooperative Oncology Group performance status of $\leq 3$; v) no history of chemotherapy or radiotherapy within 4 weeks; vi) adequate bone marrow function (neutrophil count of $1500 / \mu 1$ or more and platelet count of $\geq 100000 / \mu 1$ ), renal function (serum creatinine levels $<2.0 \mathrm{mg} / \mathrm{dl}$ ), and hepatic function (total serum bilirubin level $<3.0 \mathrm{mg} / \mathrm{dl}$, levels of aspartate aminotransferease and alanine aminotransferase less than or equal to twice the upper limits of the normal ranges); and vii) written informed consent. The study protocol was approved by the institutional review board of Saitama Medical University.

Treatment protocol. S-1 was given orally twice daily for 28 consecutive days, followed by 14 days of rest. The dose of $\mathrm{S}-1$ was $80 \mathrm{mg} /$ day for patients with a body surface area (BSA) of $<1.25 \mathrm{~m}^{2}, 100 \mathrm{mg} /$ day for those with a BSA of $1.25-1.5 \mathrm{~m}^{2}, 120 \mathrm{mg} /$ day for those with a BSA $>1.5 \mathrm{~m}^{2} . \mathrm{S}-1$ was administered at these doses, alone or in combination with $60 \mathrm{mg} / \mathrm{m}^{2}$ of cisplatin on day 8 .

CYP2A6 genotypes. Genomic DNA was extracted from $200 \mu \mathrm{l}$ peripheral blood, which had been stored at $-80^{\circ} \mathrm{C}$ until analysis, with a QIAamp Blood kit (Qiagen, Hilden, Germany). $C Y P 2 A 6^{*} 4 A$ was analyzed with the polymerase chain reaction (PCR) restriction fragment length polymorphism method described by Nakajima et al (15). CYP $2 A 6^{*} 7$ and $C Y P 2 A 6^{*} 9$ were analyzed with the allele-specific PCR-based method described previously in detail $(16,17)$. On the basis of the CYP2A6 genotype, the patients were divided into 3 groups: ${ }^{*} 1 /{ }^{*} 1$ as wild-type; ${ }^{*} 1 /{ }^{*} 4 A,{ }^{*} 1 /{ }^{*} 7$ and ${ }^{*} 1 /{ }^{*} 9$ as 1 variant allele; and ${ }^{*} 4 A I^{*} 4 A,{ }^{*} 4 A /{ }^{*} 7,{ }^{*} 4 A / 9,{ }^{*} 7 / * 7,{ }^{*} 7 / * 9$ and ${ }^{*} 9 / * 9$ as 2 variant alleles.
Plasma concentrations of CDHP, FT and 5-FU. Blood samples for PK analysis of CDHP, FT and 5-FU were obtained on the first day of treatment. Blood samples were taken immediately before $\mathrm{S}-1$ was administered and $0.5,1,2$, 4,8 and $24 \mathrm{~h}$ after the first dose of S-1. The second dose of S-1 on the first day was skipped for the PK analysis until 24 $\mathrm{h}$ after the first dose. The samples were centrifuged immediately, and the plasma was stored at $-80^{\circ} \mathrm{C}$ until analysis. The plasma concentrations of CDHP, FT and 5-FU were determined with the method of Matsushima et al (18). Briefly, FT and 5-FU were analyzed with a high-performance liquid chromatograph and CDPH was analyzed with a gas chromatography-negative ion chemical ionization mass spectrometry system.

PPK model building. The PPK analysis was performed with the Nonlinear Mixed Effect Model (NONMEM) program (double precision, version V, level 1.1) with the PREDPP library and the NM-TRAN preprocessor on IBM Intelistation. The Fortran compiler used was the Compaq Visual Fortran version 6.5. The basic parameters were clearance (CL), the distribution volumes (V), first-order absorption rate constant (Ka) and first-order elimination rate constant $(\mathrm{K})$. Since all doses were given by oral administration, the parameters $\mathrm{CL}$ and $\mathrm{V}$ were interpreted as $\mathrm{CL} / \mathrm{F}$ and $\mathrm{V} / \mathrm{F}$, respectively, where $\mathrm{F}$ is bioavailability.

A one compartment liner model with first-order absorption (PREDPP library, subroutine ADVAN2, TRANS2) with or without absorption lag time $\left(\mathrm{T}_{\mathrm{lag}}\right)$ was used to describe the PK of CDHP. When the Ka of CDHP was not fixed, the computation was not converged. Thus the Ka of CDHP was fixed at 1.2 according to the report of Comets et al (7). As the introduction of $\mathrm{T}_{\text {lag }}$ did not improve the model fitting, $\mathrm{T}_{\mathrm{lag}}$ was not included in the model.

To find an optimal model for the PK of FT, one or two compartment models with $\mathrm{Ka}$ and $\mathrm{K}$, with or without $\mathrm{T}_{\text {lag }}$, were tested. A one-compartment model was more suitable than a two-compartment model for FT. Therefore, a onecompartment liner model with first-order absorption (PREDPP library, subroutine ADVAN2 and TRANS2) was used to describe the PK of FT.

We, then described the PK of 5-FU, including FT and the inhibition of 5-FU elimination as a function of the timedependent concentration of CDHP $\left(\mathrm{C}_{\mathrm{CDHP}}\right)$ on 5-FU catabolism, using the individual $\mathrm{PK}$ parameters of the FT concentration and individual Bayesian predictions of the CDHP concentration according to the following equations:

$F U=\frac{\text { Dose } \cdot K a \cdot K_{r T}}{V_{F v}}\left\{\frac{\exp \left(-K_{r v} \cdot t\right)}{\left(K_{r T}-K_{r v}\right)\left(K a-K_{F v}\right)}+\frac{\exp \left(-K_{r T} \cdot t\right)}{\left(K_{F U}-K_{F T}\right)\left(K a-K_{F T}\right)}+\frac{\exp (-K a \cdot t)}{\left(K_{F U}-K a\right)\left(K_{r T}-K a\right)}\right\}$

where Dose is the dose of FT, $\mathrm{Ka}$ is the $\mathrm{Ka}$ of FT and $\mathrm{t}$ is the time. $\mathrm{K}=\mathrm{K}(0) /\left(1+\mathrm{C}_{\mathrm{CDHP}} / \mathrm{K}_{\mathrm{i}}\right)$, where $\mathrm{K}(0)$ is the rate constant of 5-FU elimination in the absence of CDHP and $\mathrm{K}_{\mathrm{i}}$ is the inhibition constant by CDHP.

The interindividual variability was assumed to obey a log-normal distribution and described for each parameter as follows: $\theta_{j}=\theta$. $\exp \left(\eta_{j}\right)$, where $\eta_{j}$ is the random effect for 
Table I. Patient characteristics.

\begin{tabular}{lc}
\hline Total no. of patients & 58 \\
Gender (M/F) & $37 / 21$ \\
Age in years (range) & $61(33-85)$ \\
Performance status $(0 / 1 / \geq 2)$ & $31 / 25 / 2$ \\
Creatinine (mg/dl) & $0.67(0.43-1.2)$ \\
Creatinine clearance ${ }^{\text {a }}(\mathrm{ml} / \mathrm{min})$ & $80.5(39-174)$ \\
Tumor location & \\
Stomach & 35 \\
Colon & 9 \\
Breast & 6 \\
Other & 8 \\
S-1 dose (mg/body/day) & 2 \\
80 & 26 \\
100 & 30 \\
120 & \\
Gastrectomy & 17 \\
Yes & 41 \\
No & \\
\hline
\end{tabular}

${ }^{\mathrm{a}} \mathrm{Ccr}$, Cockcroft-Gault equation.

the individual $\mathrm{j}, \theta$ is the population mean parameter and $\eta$ is a random variable with mean zero and variance $\omega^{2}$. Residual variability was described by a proportional error model: $C p_{i, j}=C p_{i, j} \exp \left(\varepsilon_{i, j}\right)$, where $C p_{i, j}$ is the $\mathrm{i}$-th model-predicted concentration for patient $\mathrm{j}$ and $\varepsilon_{\mathrm{i}, \mathrm{j}}$ denotes the residual intraindividual random error.

Demographic variables, such as the CYP2A6 genotype, patient age, gender, BSA, history of gastrectomy and creati- nine clearance (Ccr) calculated with the Cockcroft-Gault equation, were examined to determine whether these variables could account for the substantial interindividual variability observed. Demographic variables were included one at a time by stepwise selection, based on the likelihood ratio test. Potentially significant covariates were identified as those factors that, when added to the basic model individually, resulted in a decrease in the objective function of 3.84 points or more (the p-value was $<0.05$ ). The leave-one-out method or bootstrap method was applied as an internal validation of the developed model.

Toxicity evaluation. Toxicities were assessed and graded according to the National Cancer Institute Common Toxicity Criteria version 2.0. All patients who received at least 1 cycle of chemotherapy of S-1 monotherapy were assessable for toxicity. The $\chi^{2}$ test or correlation coefficient was used to assess the significance of findings; differences with a p-value $<0.05$ were considered significant.

\section{Results}

Patient characteristics. From September 2005 through September 2007, 58 Japanese patients with solid tumor were enrolled for PK analysis (Table I). Of 58 patients, 17 (29\%) patients with gastric cancer underwent gastrectomy before being enrolled in this study: 10 patients underwent total gastrectomy and 7 patients underwent partial gastrectomy. Of the 58 patients, 56 patients received S- 1 monotherapy and 2 received the combination of cisplatin and $S-1$. Two patients had Ccr of $<50 \mathrm{ml} / \mathrm{min}$.

CYP2A6 genotypes. In 1 patient the CYP2A6 genotype was not examined. The distribution of $C Y P 2 A 6$ genotypes was as follows: ${ }^{*} 1 /{ }^{*} 1$ in 12 patients $(21 \%),{ }^{*} 1 / 4 A$ in 9 patients $(16 \%),{ }^{*} 1 /{ }^{*} 7$ in 4 patients $(7 \%),{ }^{*} 1 /{ }^{*} 9$ in 10 patients $(17 \%)$, ${ }^{*} 4 A /{ }^{*} 4 A$ in 3 patients $(5 \%),{ }^{*} 4 A / 7$ in 6 patients $(10 \%),{ }^{*} 4 A / 9$ in 3 patients $(5 \%),{ }^{*} 7 / * 7$ in 4 patients $(7 \%),{ }^{*} 7 /{ }^{*} 9$ in 4 patients

Table II. Estimated parameters of population pharmcokinetic model.

\begin{tabular}{|c|c|c|c|}
\hline Compound & Parameter & Population mean & Interindividual variability $(\%)$ \\
\hline \multirow[t]{4}{*}{ CDHP } & $\mathrm{Ka}\left(\mathrm{h}^{-1}\right)$ & 1.2 & 174.6 \\
\hline & $\mathrm{CL} / \mathrm{F}(\mathrm{l} / \mathrm{h})$ & $\left(5.86+0.025^{*} \mathrm{Ccr}\right)^{*} \mathrm{BSA}$ & 22.8 \\
\hline & $\mathrm{V} / \mathrm{F}(1)$ & $42.7^{*} \mathrm{BSA}$ & 27.4 \\
\hline & Residual variability (\%) & 25.6 & \\
\hline \multirow[t]{6}{*}{ FT } & $\mathrm{Ka}\left(\mathrm{h}^{-1}\right)$ (no gastric resection) & 1.39 & 115.3 \\
\hline & $\mathrm{Ka}\left(\mathrm{h}^{-1}\right)$ (gastric resection) & $1.62 * 1.39$ & \\
\hline & $\mathrm{CL} / \mathrm{F}(\mathrm{l} / \mathrm{h})(C Y P 2 A 6:$ no/one variant allele $)$ & $2.02 * \mathrm{BSA}$ & 29.1 \\
\hline & CL/F (1/h) (CYP2A6: two variant alleles) & $0.58^{*} 2.02 * \mathrm{BSA}$ & \\
\hline & $\mathrm{V} / \mathrm{F}(1)$ & $23.5^{*} \mathrm{BSA}$ & 19.7 \\
\hline & Residual variability (\%) & 20.3 & \\
\hline \multirow[t]{3}{*}{$5-\mathrm{FU}$} & $\mathrm{K}\left(\mathrm{h}^{-1}\right)$ & $1.44 /\left(1+\mathrm{C}_{\mathrm{CDHP}} / 0.0127\right)$ & 0.0015 \\
\hline & $\mathrm{V} / \mathrm{F}(\mathrm{l})$ & 38.8 & 24.6 \\
\hline & Residual variability (\%) & 38.6 & \\
\hline
\end{tabular}


A

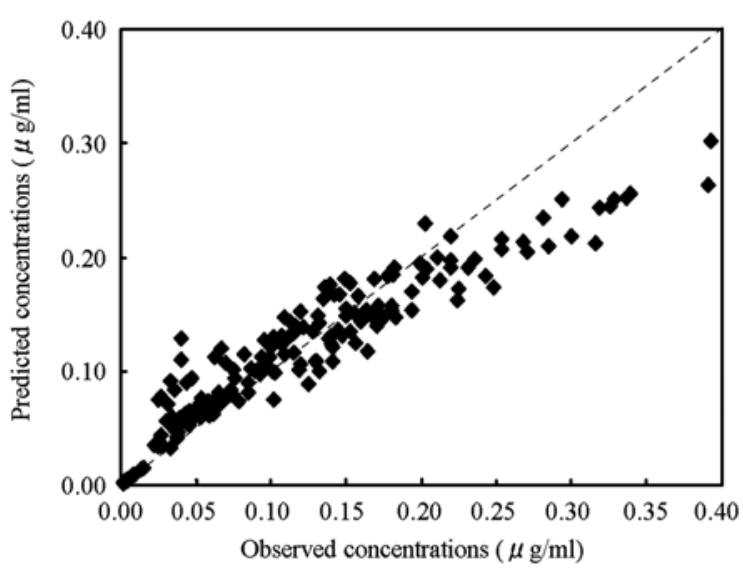

$\mathrm{C}$

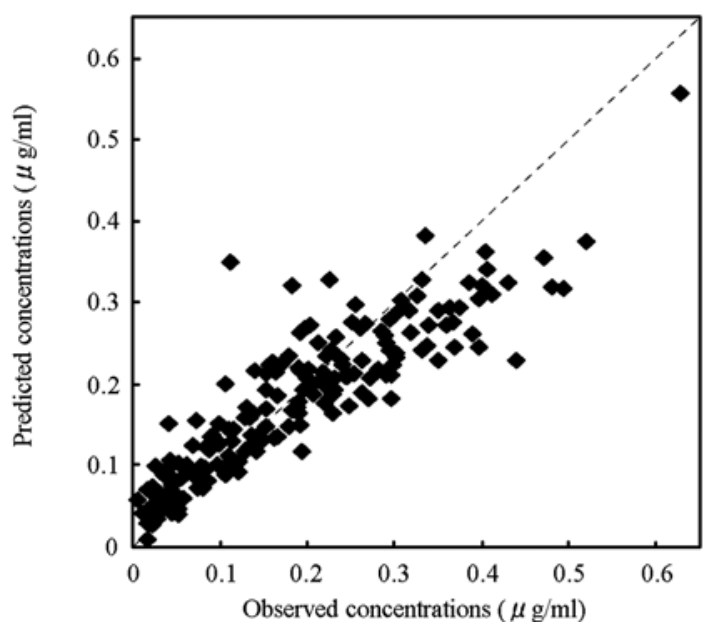

(7\%) and $* / * 9$ in 2 patients $(3 \%)$. Therefore, $21 \%$ of patients had the wild-type genotype, $40 \%$ had 1 variant allele and $38 \%$ had 2 variant alleles.

$P K$ analysis of $C D H P$. The $C Y P 2 A 6$ genotype, patient age, gender and the history of gastrectomy did not affect to the individual $\mathrm{CL}, \mathrm{V}$ and $\mathrm{Ka}$ of $\mathrm{CDHP}$. The individual $\mathrm{CL}$ of CDHP was proportional to the Ccr. In addition, the individual CL and V of CDHP were proportional to the BSA. The estimated population mean and variance of the PK parameters of CDHP are shown in Table II. The individual Bayesian predictions of CDHP concentrations based on the developed PPK model were in good agreement with the observed concentrations data (Fig. 1A), with residual errors uniformly distributed.

$P K$ analysis of FT. The patient age, gender and Ccr did not affect to the individual CL, $\mathrm{V}$ and $\mathrm{Ka}$ of FT. The individual $\mathrm{CL}$ and $\mathrm{V}$ of FT were proportional to the BSA. In patients with 2 variant alleles of $C Y P 2 A 6$ genotype, the $C L$ of FT was $58 \%$ less than that in patients with wild-type or 1 variant allele of CYP2A6 (Fig. 2A). In addition, in patients with a history of gastrectomy, the Ka of FT was $62 \%$ higher than in patients without a history of gastrectomy (Fig. 2B). Moreover, in patients with a history of total gastrectomy, the Ka of FT was higher than in patients with a history of partial gastrectomy,

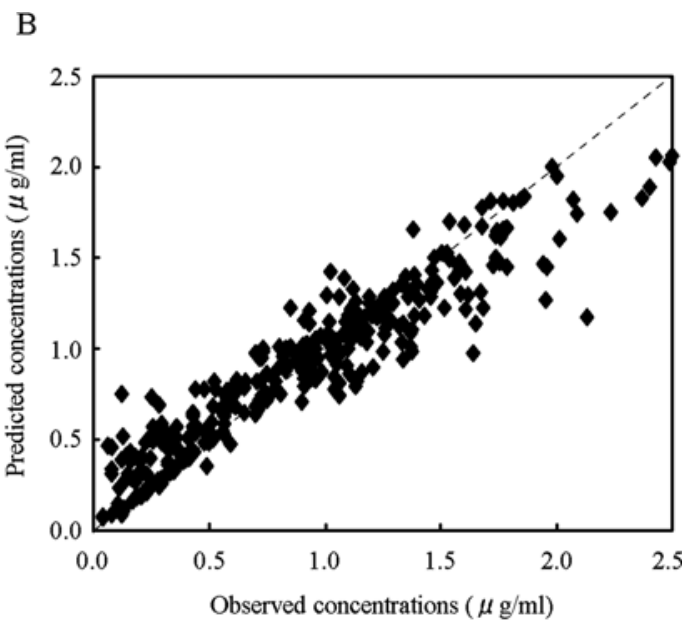

Figure 1. A plot of the Bayesian predicted concentrations versus the observed concentrations for 3 compounds: (A) 5-chloro-2,4-dihydroxypyridine (CDHP), (B) tegafur (FT) and (C) 5-fluorouracil (5-FU). The line in the panel represents the line of unity.

although the highest Ka of FT was in patient with the history of partial gastrectomy. The estimated population mean and variance of the PK parameters of FT are shown in Table II. The individual Bayesian predictions of FT concentrations based on the developed PPK model were in good agreement with the observed concentrations data (Fig. 1B), with residual errors uniformly distributed.

$P K$ analysis of 5-FU. One compartmental structural kinetic model was suitable to describe the current 5-FU data. The inhibitory effect of $\mathrm{C}_{\mathrm{CDHP}}$ on 5-FU catabolism was modeled according to the following equation derived from the competitive enzymatic inhibition model: $\mathrm{K}=\mathrm{K}(0) /\left(1+\mathrm{C}_{\mathrm{CDHP}} / \mathrm{K}_{\mathrm{i}}\right)$. The estimated population mean and variance of the PK parameters of 5-FU are shown in Table II. The individual Bayesian predictions of 5-FU concentrations based on the developed PPK model were in good agreement with the observed concentrations data (Fig. 1C), with residual errors uniformly distributed.

Model validation. Results of the validation $>95 \%$ were converged. The differences between the final model estimate and validation mean were $<19.3 \%$. The results of validation indicated reliability and robustness of the parameter estimates. Thus, our population pharmacokinetic model is acceptable. 


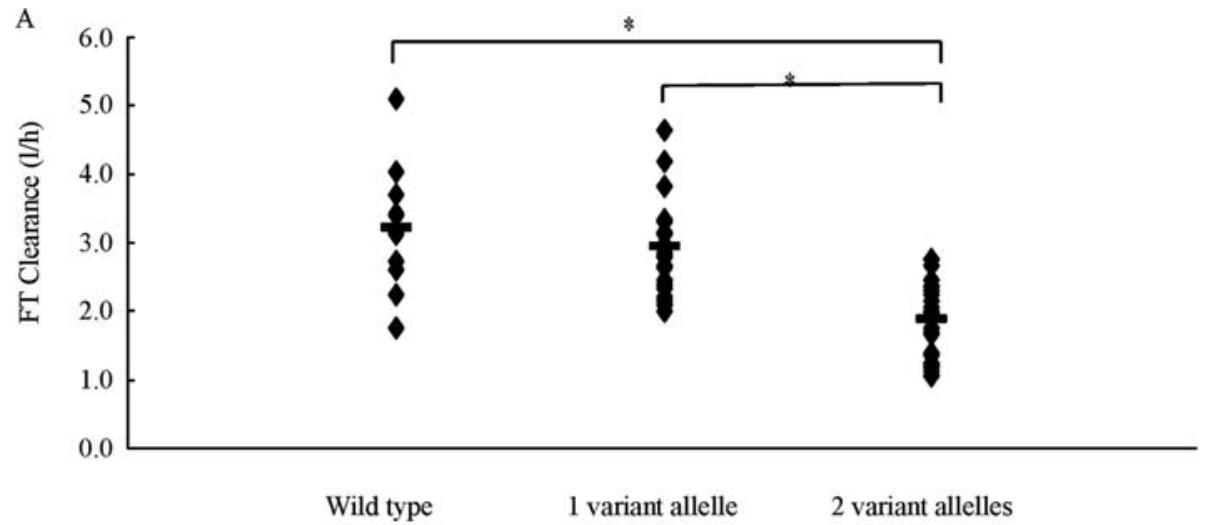

CYP2A6 gene

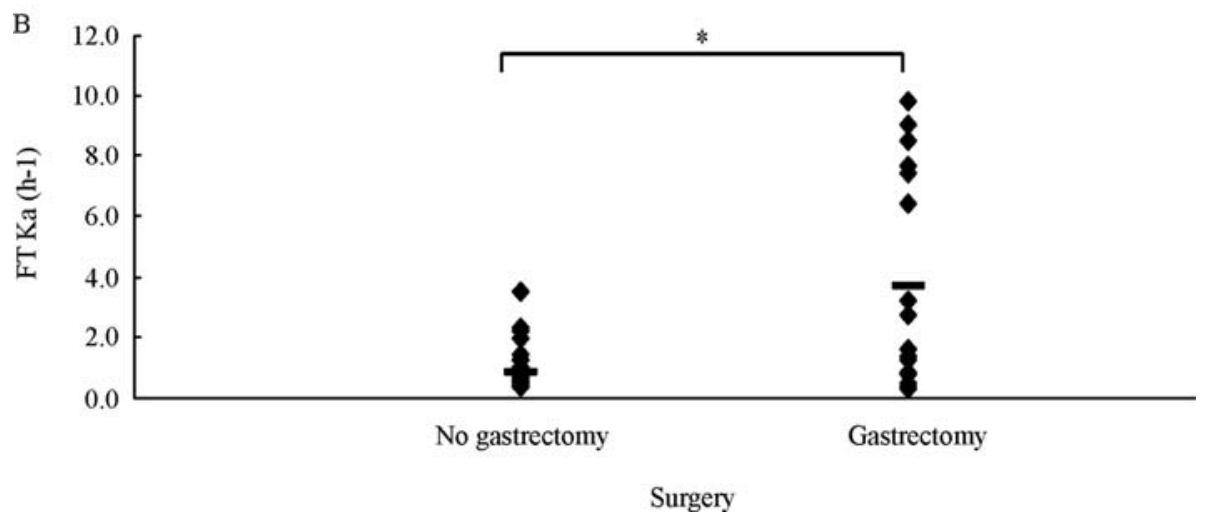

Figure 2. (A) The relationship between the CYP2A6 genotype and crearance (CL) of tegafur (FT). (B) The relationship between a history of gastrectomy and the first-order absorption rate constant (Ka) of FT.

A

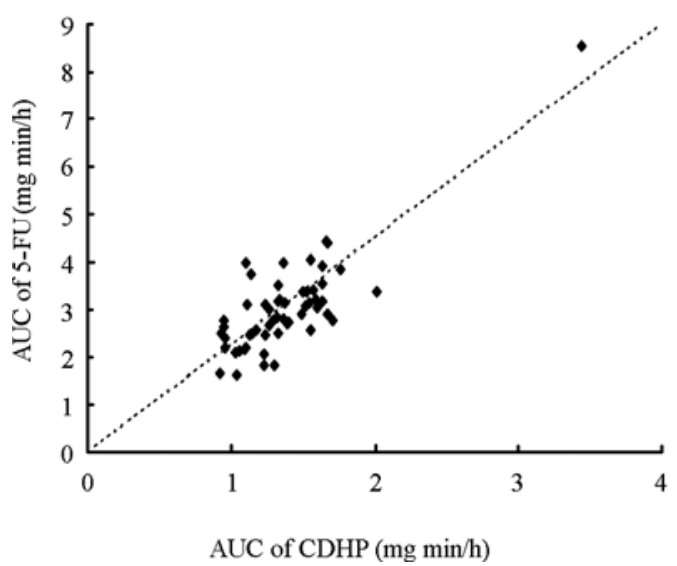

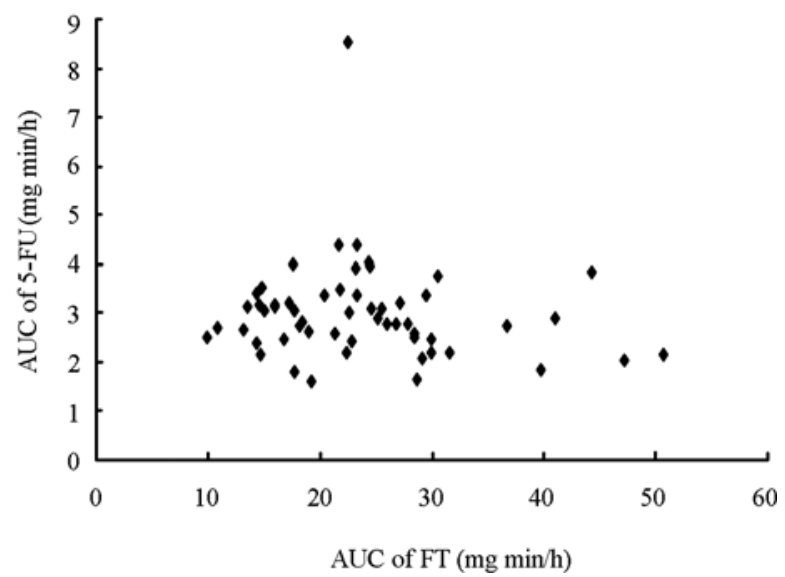

Figure 3. (A) The area under the plasma concentration-time curve (AUC) of 5-fluorouracil (5-FU) and AUC of 5-chloro-2,4-dihydroxypyridine (CDHP). (B) The AUC of 5-FU and the AUC of tegafur (FT).

Factors influencing the AUC of 5-FU. The AUC of 5-FU based on this PPK model correlated with the AUC of CDHP $(\mathrm{p}<0.0001$; Fig. 3A), whereas the AUC of 5-FU did not correlate with the AUC of FT ( $\mathrm{p}=0.37$; Fig. 3B). Therefore, the $C Y P 2 A 6$ genotype and the history of gastrectomy did not affect the AUC or Cmax of 5-FU.
Exposure-toxicity analysis for 5-FU. Of the 58 patients, 56 patients administered S-1 monotherapy were analyzed for the relationship of toxicity and the AUC or Cmax of 5-FU on the basis of the developed PPK model. Table III lists the maxmum toxicities experienced during S-1 administration. The most frequent toxicity was myelosuppression, especially 
Table III. Toxicity of S-1 monotherapy.

\begin{tabular}{|c|c|c|c|c|c|}
\hline \multirow[b]{2}{*}{ Toxicity } & \multicolumn{5}{|c|}{ National Cancer Institute-Common Toxicity Criteria Grade } \\
\hline & 1 & 2 & 3 & 4 & 3 or $4(\%)$ \\
\hline Leukopenia & 5 & 3 & 5 & 0 & 8.9 \\
\hline Neutropenia & 7 & 4 & 3 & 1 & 7.1 \\
\hline Thrombocytopenia & 6 & 1 & 1 & 2 & 5.4 \\
\hline Anemia & 20 & 18 & 10 & 1 & 19.6 \\
\hline Nausea & 14 & 7 & 5 & 0 & 8.9 \\
\hline Diarrhea & 10 & 4 & 1 & 1 & 5.4 \\
\hline Anorexia & 9 & 5 & 7 & 0 & 12.5 \\
\hline Infection & 7 & 5 & 5 & 0 & 8.9 \\
\hline Elevation of serum creatinine & 0 & 0 & 1 & 0 & 1.8 \\
\hline Elevation of transaminase & 9 & 0 & 3 & 1 & 7.1 \\
\hline Fatigue & 14 & 11 & 8 & 1 & 16.1 \\
\hline
\end{tabular}
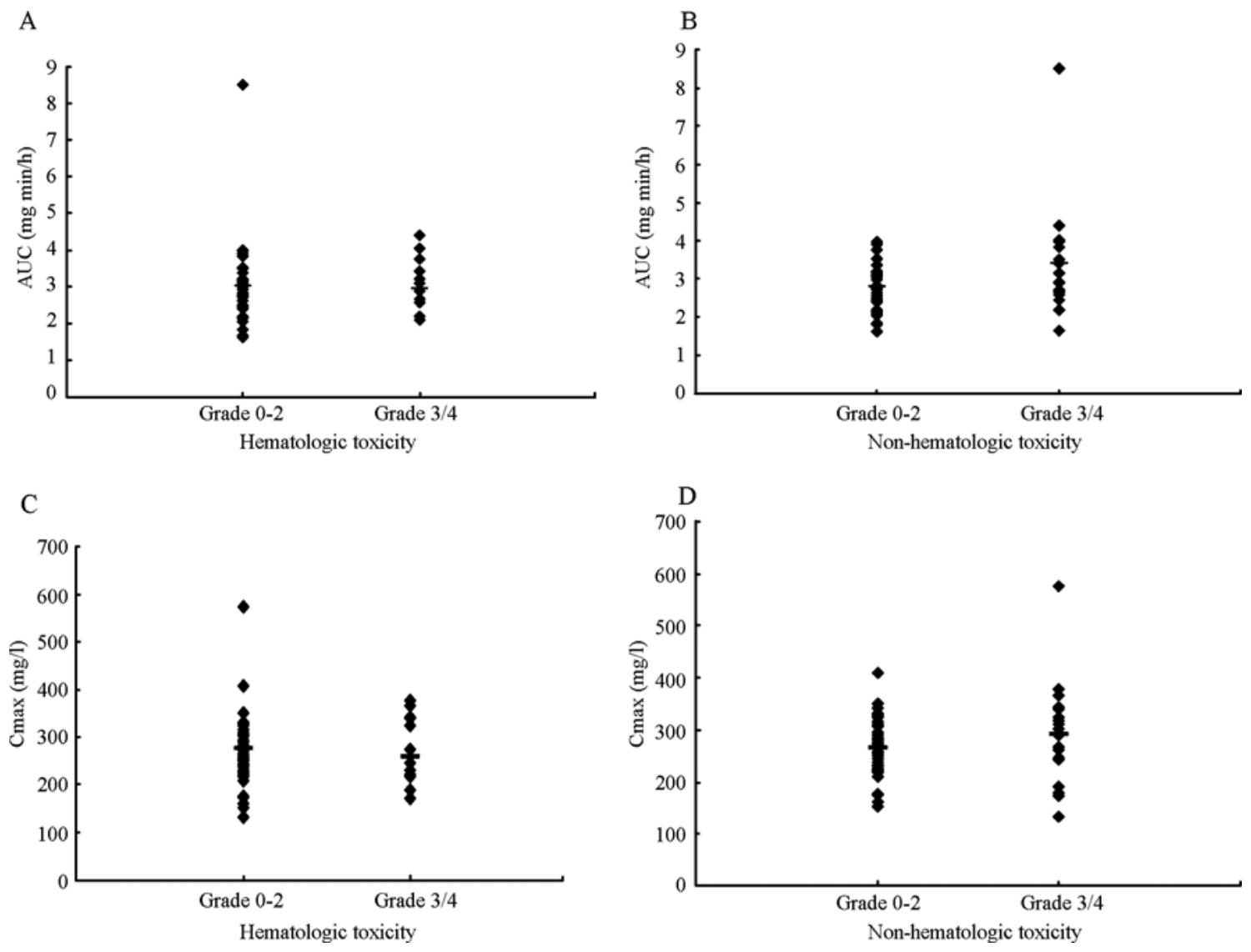

Figure 4. (A and B) Relationship between the individual the area under the plasma concentration-time curve (AUC) of 5-FU and toxicity: (A) hematologic toxicity, (B) non-hematologic toxicity. (C and D) Relationship between the individual the individual maximum concentrations (Cmax) values of 5-FU and toxicity: (C) hematologic toxicity, (D) non-hematologic toxicity.

anemia. The most frequent non-hematologic toxicity was fatigue, followed by gastrointestinal toxicity. The individual AUC or Cmax of 5-FU did not differ significantly between patients with grade 3 or 4 hematologic or non-hematologic toxicities and patients with grade $0-2$ toxicities (Fig. 4). On the other hand, a patient with colon cancer and 1 variant allele of CYP2A6 gene died 5 days after starting S-1 administration because of severe stomatitis and diarrhea, and as a consequence dehydration. The pretreatment serum creatinine and Ccr of this patient were 1.2 and $45 \mathrm{ml} / \mathrm{min}$, respectively. In this patient, the plasma concentration, AUC and Cmax of 5-FU were the highest of all enrolled patients. 


\section{Discussion}

We developed PPK model of S-1 including the CYP2A6 genotype and investigated the influence of the $C Y P 2 A 6$ genotype on PK parameters of S-1 because the biotransformation of FT to 5-FU is catalyzed by the hepatic drug-metabolizing enzyme CYP2A6 (12). In our study, the CL of FT was 58\% lower in patients with 2 variant alleles of $C Y P 2 A 6$ than in patients with the wild-type genotype or 1 variant allele. Therefore, our results show that the presence of 2 variant alleles of $C Y P 2 A 6$ is associated with an interindividual variation of $C Y P 2 A 6$ activity.

We included gastrectomy in the PPK model of FT because after gastrectomy the retentive function of the stomach is lost or reduced, and FT rapidly enters the small intestine after ingestion and is absorbed there. In the present study, in patients with a history of gastrectomy, the Ka of FT was $62 \%$ higher than in patients without a history of gastrectomy. Moreover, in patients with a history of total gastrectomy, the Ka of FT was higher than in patients with a history of partial gastrectomy. Kochi et al have reported (19) that the Cmax and AUC of FT is higher in patients with a history of total gastrectomy but not in patients with a history of partial gastrectomy.

In the present study, the AUC of 5-FU did not correlate with the AUC of FT, whereas the AUC of 5-FU correlated with the AUC of CDHP. Therefore, neither the CYP2A6 genotype nor a history of gastrectomy had an effect on the AUC of 5-FU. This finding indicates that the CL of FT is affected by $C Y P 2 A 6$ genotype and the absorption rate of FT is increased by gastrectomy, but that the amount of the active metabolite 5-FU does not change. Our findings were contrary to those of a previous study, which Cmax of 5-FU were significantly lower in patients with the $C Y P 2 A 6^{*} 4 C$ allele than in patients without the $C Y P 2 A 6^{*} 4 C$ allele (20). Until now, the relationship between the CL of FT to 5-FU and the AUC of 5-FU has been controversial $(7,8)$. Ajani et al have reported that the CL of FT to 5-FU is higher in whites than in Asians; thus, 5-FU achieves a higher AUC in whites than in Asians (8). On the other hand, Comets et al have reported that the CL of FT to 5-FU is higher in whites than in Asians, but the AUC of 5-FU differs only slightly between whites and Asians (7). Because $90 \%$ of administered 5-FU is rapidly catabolized into inactive metabolites by DPD in the liver, the plasma concentration of 5-FU cannot increase without an inhibitory effect of CDHP on DPD $(21,22)$. A previous study found that the concentration of 5-FU is more strongly correlated with the concentration of CDHP than with the concentration of FT (19). Therefore, we believe that 5-FU exposure is affected primarily by the concentration of CDHP rather than the biotransformation of FT.

Thus, the inhibitory effect of CDHP on DPD should be considered for the PPK modeling of S-1. We performed a separate analysis of the PK of CDHP and included the timedependent plasma concentration of CDHP with competitive enzymatic inhibition for the PK analysis of 5-FU. Because the PK of CDHP is independent of the PK of FT, and CDHP acts through an inhibitor on 5-FU catabolism, the present sequential fitting approach is appropriate. We estimated the $\mathrm{K}_{\mathrm{i}}$ to be $0.0127 \mathrm{mg} / \mathrm{l}$. The $\mathrm{K}_{\mathrm{i}}$ has been reported $0.04 \mathrm{mg} / \mathrm{l}$ in Western cancer patients (7), and in vitro the inhibition constant of CDHP has been reported to be $0.05 \mathrm{mg} / \mathrm{l}$ (23). Therefore, our estimated $\mathrm{K}_{\mathrm{i}}$ is comparable to previously published clinical and preclinical data.

In the present study, the individual CL of CDHP was proportional to Ccr. Because most CDHP is excreted into the urine in an unchanged form, renal function is an important factor in the plasma CL of CDHP (8). The CL of CDHP is prolonged in the presence of renal impairment, leading to a longer half-life and higher AUC of 5-FU (22). As a result, renal impairment may lead to severe adverse events. A nationwide survey of S-1 in Japanese patients with advanced gastric cancer found that hematological toxicities of grade 3 or worse occurred significantly more often in patients with impaired renal function (24). Additionally, Kong et al reported patients with the lower Ccr level developed the higher incidence of grade 2 or more nausea, vomiting, anorexia, fatigue, or other gastrointestinal toxicity (25). We cannot make any definitive conclusions about patients with impaired renal function in this study, because only 2 patients had a Ccr of less than $50 \mathrm{ml} /$ min before treatment. However, 1 patient who died of the toxic effects of S-1 had a pretreatment Ccr of $45 \mathrm{ml} / \mathrm{min}$. Therefore, administering S-1 to patients with impaired renal function may need individualized dosing and PK monitoring.

In the present study, a patient who died from the toxic effects of S-1 had the highest plasma concentration, AUC and $\mathrm{Cmax}$ of 5-FU of all enrolled patients. However, the individual AUC and Cmax of 5-FU did not differ significantly between patients with grade 3 or 4 toxicities and patients with grade 0-2 toxicities. Several reasons for this lack of difference can be considered. First, the pretreatment characteristics of the patients in this study were variable. These variable pretreatment characteristics and laboratory values may have influenced the frequency and severity of the toxicity as well as the PK profiles. Second, we examined the PPK of the only first dose of S-1, although S-1 was given for consecutive 28 days. The relation between the AUC of 5-FU and toxicity has been controversial $(8-11,20)$. Some previous studies have reported that the toxicity and the antitumor activity of 5-FU are not correlated with the AUC or Cmax of 5 -FU $(9,20)$. On the other hand, some studies have reported that the toxic effects, such as the severe diarrhea of 5-FU, are correlated with the AUC of 5-FU $(8,10,11)$.

In conclusion, we developed the PPK model of S-1 including the $C Y P 2 A 6$ genotype in Japanese patients with advanced cancer. The presence of 2 variant alleles of $C Y P 2 A 6$ decreased the CL of FT by $58 \%$ compared with the wild-type or 1 variant allele. However, the AUC of 5-FU was correlated with the AUC of CDHP but was not correlated with the AUC of FT. Therefore, the CYP2A6 genotype did not affect the AUC of 5-FU. Further large prospective studies including measurement of CYP2A6 genotype and renal function are needed to confirm our findings and clarify the correlation between the PK of 5-FU and the response or toxicity.

\section{References}

1. Shirasaka T, Nakano K, Takechi T, et al: Antitumor activity of $1 \mathrm{M}$ tegarur- $0.4 \mathrm{M}$ 5-chloro-2,4-dihydroxypyridine-1 M potassium oxonate (S-1) against human colon carcinoma orthotopically implanted into nude rats. Cancer Res 56: 26022606, 1996. 
2. Shirasaka T, Shimamoto Y and Fukushima M: Inhibition by oxonic acid of gastrointestinal toxicity of 5-fluorouracil without loss of its antitumor activity in rats. Cancer Res 53: 4004-4009, 1993.

3. Koizumi W, Narahara H, Hara T, et al: S-1 plus cisplatin versus $\mathrm{S}-1$ alone for first-line treatment of advanced gastric cancer (SPIRITS trial): a phase III trial. Lancet Oncol 9: 215-221, 2008.

4. Goto A, Yamada Y, Yasui K, et al: Phase II study of combination therapy with S-1 and irinotecan in patients with advanced colorectal cancer. Ann Oncol 17: 968-973, 2006.

5. Kawahara M, Furuse K, Segawa Y, et al: Phase II study of S-1, a novel oral fluorouracil, in advanced non-small cell lung cancer. Br J Cancer 85: 939-943, 2001.

6. Evans WE and Mcleod HL: Pharmacogenomics - drug disposition, drug targets and side effects. N Eng J Med 348: 538-549, 2003.

7. Comets E, Ikeda K, Hoff P, Fumoleau P, Wanders J and Tanigawara Y: Comparison of the pharmacokinetics of S-1, an oral anticancer agent, in Western and Japanese patients. J Pharmacokin Pharmacodyn 30: 257-283, 2003.

8. Ajani JA, Faust J, Ikeda K, et al: Phase I pharmacokinetic study of S-1 plus cisplatin in patients with advanced gastric carcinoma. J Clin Oncol 23: 6957-6965, 2005.

9. Hirata K, Horikoshi N, Aiba K, et al: Pharmacokinetic study of S-1, a novel oral fluorouracil antitumor drug. Clin Cancer Res 5: 2000-2005, 1999.

10. Van Groeningen CJ, Peter GJ, Schornagel JH, et al: Phase I clinical and pharmacokinetic study of oral S-1 in patients with advanced solid tumors. J Clin Oncol 18: 2772-2779, 2000.

11. Hoff PM, Saad ED, Ajani JA, et al: Phase I study with pharmacokinetics of S-1 on an oral daily schedule for 28 days in patients with solid tumors. Clin Cancer Res 9: 134-142, 2003.

12. Ikeda K, Yoshisue K, Matsushima E, et al: Bioactivation of tegafur to 5-fluorouracil is catalyzed by cytochrome P450 2A6 in human liver microsomes in vitro. Clin Cancer Res 6: 4409-4415, 2000.

13. Fujita K: Cytochrome P450 and anticancer drugs. Curr Drug Metab 7: 23-37, 2006

14. Nakajima M, Fukami T, Yamanaka H, et al: Comprehensive evaluation of variability in nicotine metabolism and CYP2A6 polymorphic alleles in four ethnic populations. Clin Pharmacol Ther 80: 282-297, 2006.

15. Nakajima M, Yoshida R, Fukami T, McLeod HL and Yokoi T: Novel human CYP2A6 alleles confound gene deletion analysis. FEBS Lett 569: 75-81, 2004.
16. Ariyoshi N, Sawamura Y and Kamataki T: A novel single nucleotide polymorphism altering stability and activity of CYP2A6. Biochem Biophys Res Commun 281: 810-814, 2001.

17. Yoshida R, Nakajima M, Nishimura K, Tokudome S, Kwon JT and Yokoi T: Effects of polymorphism in promoter region of human $C Y P 2 A 6$ gene $\left(C Y P 2 A 6^{*} 9\right)$ on expression level of messenger ribonucleic acid and enzymatic activity in vivo and in vitro. Clin Pharmacol Ther 74: 69-76, 2003.

18. Matsushima E, Yoshida K, Kitamura R and Yoshida KI: Determination of S-1 (combined drug of tegafur, 5-chloro-2,4dihydroxy-pyridine and potassium oxonate) and 5-fluorouracil in human plasma and urine using high-performance liquid chromatography and gas chromatography-negative iron chemical ionization mass spectrometry. J Chromatogr B 691: 95-104, 1997.

19. Kochi M, Fujii M, Kanamori N, Kaiga T, Aizaki K, Takahashi T and Takayama T: Effect of gastrectomy on the pharmacokinetics of S-1, an oral fluoropyrimidine, in respectable gastric cancer patients. Cancer Chemother Pharmacol 60: 693-701, 2007.

20. Kaida Y, Inui N, Suda T, Nakamura H, Watanabe H and Chida K: The CYP2A6 4 allele is determinant of S-1 pharmacokinetics in Japanese patients with non-small cell lung cancer. Clin Pharmacol Ther 83: 589-594, 2008.

21. Tsuruoka Y, Kamano T, Kitajima M, et al: Effect of gastrectomy on the pharmacokinetics of 5-fluorouracil and gimeracil after oral administration of S-1. Anticancer Drugs 17: 393-399, 2006.

22. Ikeda M, Furukawa H, Imamura H, et al: Pharmacokinetic study of S-1, a novel oral fluorouracil antitumor agent in animal model and in patients with impaired renal function. Cancer Chemother Pharmacol 50: 25-32, 2002.

23. Tatsumi K, Fukushima M, Shirasaka T and Fujii S: Inhibitory effect of pyrimidine, barbituric acid and pyridine derivatives of 5-fluorouracil degradation in rat liver extracts. Jpn J Cancer Res 78: 748-755, 1987.

24. Nagashima F, Ohtsu A, Yoshida S and Ito K: Japanese nationwide post-marketing survey of S-1 in patients with advanced gastric cancer. Gastric Cancer 8: 6-11, 2005

25. Kong SY, Lim HS, Nam BH, et al: Association of CYP2A6 polymorphisms with S-1 plus docetaxle therapy outcomes in metastatic gastric cancer. Pharmacogenomics 10: 1147-1155, 2009. 